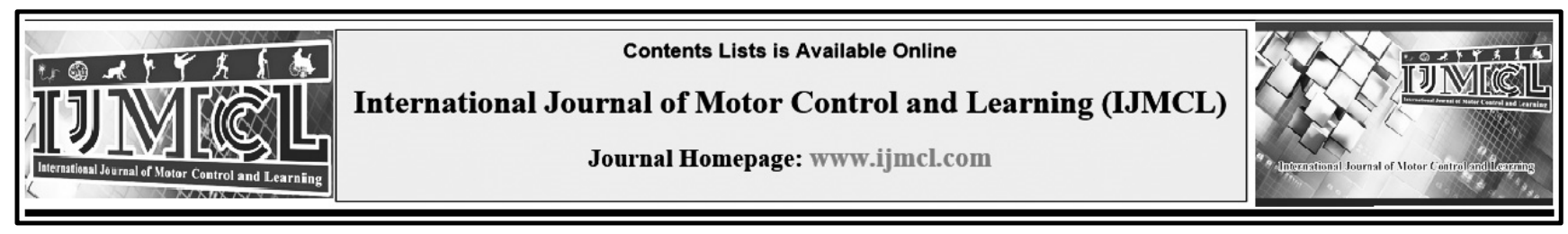

\title{
Visual Search: The Effect of Quiet Eye Training
}

\author{
Mina Keshavarz ${ }^{a}$, Jalal Dehghanizade ${ }^{b *}$ \\ a $\mathrm{PhD}$ Student in Motor Behavior, Urmia University, Urmia, Iran \\ ${ }^{\mathrm{b}}$ Assistant Professor of Motor Behavior, Urmia University, Urmia, Iran \\ * Corresponding E-mail: jalal.dehghanizade@yahoo.com
}

Received: 2021/02/07 | Accepted: 2021/04/18 | Published: 2021/05/05

\section{DEAR EDITOR}

Over the years, the acquisition of motor skills has been considered a necessity in the field of sports science. Studies in the field of motor behavior have been planned and conducted over the years to find appropriate scientific and practical theories. Although in each course theories were presented to properly justify the acquisition of motor skills, the shortcomings of each idea and theory led researchers to provide more comprehensive models that align the principles of exercise science with the actual practice environment to determine how the learner acquires motor skills. What is your current view of motor behavior and how does your inference base your teaching and coaching methods? Therefore, it is necessary to know which model offers more scientific and practical instructions for organizing training fields, providing information for the learner, using visual representation and other important tasks. As a result, many traditional theories have been developed to explain a small group of related actions (Newell, 1989). For example, open-loop theories are adapted for fast throwing motions; Closed-loop theory examines slow and precise movements, and schema theory is proposed to explain the learning of discrete movements. Traditional approaches to skill acquisition have not provided a single theory for learning (Anson, G., Elliott, D., \& Davids, K, 2005). In contrast, new theories such as dynamic systems have more stably explained motor acquisition.
Dynamic systems theory emphasizes the need to understand natural phenomena as a system with high interaction between components (Clarke, D., \& Crossland, J, 1985). The view of dynamic systems provides a very good justification and basis for the study of human behavior. In other words, dynamic systems believe in coordination, complexity, self-organization and information constraints during the acquisition of motor skills (Davids, Button and Bennett, 2008). Over the past century, the issue of how humans learn to control and coordinate their movements has received much attention from scientists and has led to various theories; However, there is still debate as to which theory is more correct (Vickers, 2011).

In recent years, much research has been done on the components of controlling visual behavior related to motor tasks. A particular strategy among these studies is the quiet eye, which has been shown to be required for high levels of motor skills and performance (Vickers, 1996); In fact, accuracy and expertise improve with increasing quiet eye duration (Vickers, 2007). In fact, the quiet eye is a perceptual skill that is associated with expertise and optimal performance. The benefits of this phenomenon have been observed in a range of motor tasks. Quiet eye training emphasizes the place and duration of eye stabilization during the skill acquisition process.

In many sports, performers have to make quick decisions in an ever-changing and complex 
environment. For example, players must act on the information provided by the ball, teammates and opponents. These decisions must be made under the pressure of opponents who are trying to limit the space and time available for execution. In such background, in order to function effectively, players must focus only on relevant and relevant sources of information. So, recognizing when and where to look is the most important aspect of skillful performance. Sometimes it has been found that the pattern of skillful vision search is not done randomly, but is based on intentional perceptual strategies (Yoshikawa, N., Nittono, H., \& Masaki, H, 2020). Eye movements through the search strategy enable the controller and the performer to use the time available to analyze the display more effectively. Visual stabilization allows the performer to stabilize an important area of the show, such as a ball or player, in central vision, and this allows for more detailed processing. The more information that has to be processed, the longer it takes to establish itself (Just, \& Carpenter, 1976).

It should be noted that research on the quiet eye in the country is very limited, which requires further investigation in different groups and communities. It should also be noted that it is necessary to study the quiet eye and practice the quiet eye with other movement training in most sports and different ages to finally be a comprehensive program for different groups to increase the duration of the quiet eye and subsequently motor function, design and implementation.

\section{Reference}

1. Anson, G., Elliott, D., \& Davids, K. (2005). Information processing and constraints-based views.

2. Clarke, D., \& Crossland, J. (1985). Action systems: An introduction to the analysis of complex behaviour. London: Methuen.

3. Davids K, Button C, Bennett S. (2008). Dynamics of Skill Acquisition: A Constraints-led Approach: Human Kinetics.

4. Davids, K., Bennett, S., \& Newell, K.M. (Eds.). (2006). Movement system variability. Champaign, IL: Human Kinetics.

5. Just, M.A. and Carpenter, P.A. (1976) 'Eye fixations and cognitive processes', Cognitive Psychology 8:441-80.

6. Yoshikawa, N., Nittono, H., \& Masaki, H. (2020). Effects of Viewing Cute Pictures on Quiet Eye Duration and Fine Motor Task Performance. Frontiers in Psychology, 11, 1565.

7. Newell, K.M., \& Slifkin, A.B. (1998). The nature of movement variability. In J.P. Piek (Ed.), Motor behavior and human skill: A multidisciplinary perspective (pp. 143-160). Champaign, IL: Human Kinetics.

8. Vickers JN. (2007). Perception, cognition and decision training: the quiet eye in action. Champaign: Human Kinetics.

9. Vickers, J. N. (1996). Visual control when aiming at a far target. Journal of Experimental Psychology: Human Perception and Performance, 22, 342-354.

10. Vickers, J. N. (2011). Mind over muscle: the role of gaze control, spatial cognition, and the quiet eye in motor expertise. Cognitive Processing, 12(3), 219-222. 